\title{
REENGENHARIA E ESTRESSE EM UNIDADES DE INFORMAÇÃo
}

\author{
Silas Marques de Oliveira
}

\begin{abstract}
Resumo
A habilidade do indivíduo de lidar com estresse no trabalho tem recebido comparativamente pouca atenção pelos pesquisadores da área de ciência da informação. Uma revisão da literatura pertinente revelou apenas alguns trabalho sobre este tópico, e a maioria enfatiza os resultados negativos e as consequências psicológicas devastadoras infligidas sobre o indivíduo através de eventos estressantes. Poucos trabalhos abordam intervenções que evitariam a ocorrência de situações estressantes, o que por sua vez levaria a resultados positivos manifestados através da melhoria do desempenho e aumento da produtividade. Sendo as unidades de informação agências de prestação de serviços, a estrutura rígida, hierárquica, funcional, não condiz com a natureza de suas atividades, provocando, assim, estresse em seu corpo de pessoal. Uma reengenharia em sua estrutura visando compatibilizá-la com sua principal função eliminará focos de tensão, evitando estresse e conseqüentemente melhorando o ambiente de trabalho propiciando, assim, um melhor aproveitamento do potencial do pessoal.
\end{abstract}

\section{Palavras-Chave}

Stress - Trabalho - Unidades de Informação

\section{INTRODUÇÃO}

Um conceito amplamente difundido na literatura administrativa e psicológica, estresse e gerenciamento de estresse tem sido, comparativamente a outras áreas, quase que negligenciado no mundo da ciência da informação. O aumento da demanda por informação por todos os setores da sociedade concomitantemente com uma diminuição do corpo de pessoal, recursos limitados, falta de conhecimento sobre administração de pessoal por administradores de unidades de informação, e a necessidade de acompanhar os rápidos desenvolvimentos dos recursos tecnológicos, estão agindo como fontes de estresse para os funcionários destas organizações, e tornando-os tão suscetíveis aos efeitos maléficos de estresse quanto qualquer outro grupo profissional.

Este estudo se propõe a identificar os principais enfoques abrangidos pela literatura da área e sugerir formas, através da reengenharia da estrutura das unidades de informação, de evitar estresse entre seus profissionais e trabalhadores.

No linguajar do dia-a-dia, "estresse" é compreendido de formas variadas, dificuldade diante de uma adversidade, uma força ou pressão 
exercida sobre uma pessoa, uma tensão mental ou nervosa. Uma definição muito citada na literatura é a oferecida por SEYLE (1:1) o fundador da pesquisa sobre estresse: "Estresse é a resposta não específica do corpo sob qualquer pressão." Uma definição mais detalhada é empregada por BERKHOUT (2:407). Ele define estresse como sendo "uma reação de um ser vivo às condições hostis em seu ambiente imediato, onde o ambiente é constituído por fatores internos psíquicos e fisiológicos assim como pelos fatores externos tradicionais."

Dois conceitos são distinguidos por estas definições. A noção da existência de um ambiente e a interação do indivíduo com este ambiente. Este estudo se limitará a discutir situações estressantes no ambiente de unidades de informação, mais especificamente, apresentará causas, estratégias de adaptação e técnicas de prevenção. Irá demonstrar que muito embora estratégias de adaptação sejam essenciais, apenas técnicas de prevenção estimularão um melhor rendimento e produtividade.

A maneira como um indivíduo reage ou se adapta a uma situação de adversidade grandemente depende de sua avaliação cognitiva com respeito à situação existente. Portanto, nem todos respondem da mesma forma diante de uma situação de risco ou desconforto. As reações variam dependendo da personalidade, experiência prévia e suscetibilidade a estresse, bem como se o indivíduo possui um alto grau de autosuficiência ou se ele é caracterizado por atitude de desamparo. BUBE $(3: 7)$ afirma que "cada pessoa recebe, interpreta, e reage a uma situação estressante de formas singulares, dependendo do significado a ela concedida, provindo de seu desenvolvimento, experiências atuais e nível cultural."

GROSSER (4) observa que as pessoas que exibem as seguintes características tendem a se adaptarem mal quando confrontadas com eventos estressantes: uma pessoa com um alto grau de ansiedade, intolerância com relação a situações ambíguas, necessidade de segurança e alto desempenho, e introvertidas. FREUDENBERGER E RICHELSON (5:249) afirmam que "os profissionais que determinam padrões de desempenho elevados para si mesmos, que exigem muito de si e de seus subalternos, que tomam para si inúmeras funções e atividades sem solicitar ajuda ou delegar, que trabalham sempre mais e mais tentando atingir sua próprias metas são aqueles que devem ser monitorados para a existência de sintomas de estresse."

Pode-se presumir, portanto, que quão maior for a intensidade ou duração de um "estressor" específico, e quanto mais "estressores" existirem simultaneamente, mais intenso serão os efeitos psicológicos se o indivíduo não lidar com a situação de forma adequada.

\section{FONTES DE ESTRESSE}

Uma reação tópica de estresse pode ser provocada por uma variedade de "estressores" físicos, ambientais e psicossociais. Os fatores usualmente apresentados e discutidos na literatura incluem: dieta; hereditariedade; estado geral de saúde; fatores de personalidade, apoio social; sistema de valores; e habilidades de adaptação.

Três pesquisas importantes relatadas na literatura de ciência da informação apresentam as principais fontes de estresse entre os bibliotecários. OLSGAARD \& SUMMERS (6) entrevistaram 51 diretores e 108 professores de escolas de biblioteconomia e ciência da informação e compararam as causas de estresse em cada grupo. Em um estudo mais recente, HODGES (7:754) relatou que "existem inúmeros fatores dentro de unidades de informação que podem afetar o nível de estresse: comunicação, retroalimentação, mudanças, influência e poder, orçamento e recursos disponíveis, autonomia e estilo gerencial." Depois de analisar 850 entrevistas com profissionais e auxiliares de bibliotecas em todo o país (USA), BUNGE (8) concluiu que as principais causas de estresse entre este grupo de profissionais são: trabalho em demasia, principalmente no que diz respeito a novos projetos sendo desenvolvidos sem a diminuição da carga regular; falta de apreciação por parte dos superiores; tarefas desestimulantes e trabalho durante os fins de semana.

A literatura analisada neste estudo revelou mais de 50 (cinquenta) variáveis diferentes que podem levar a um estado de estresse. Estas variáveis são consideradas como sendo estressores que se não forem eliminados ou evitados por estratégias de intervenção ou se não forem lidados adaptativamente, provocarão efeitos físicos e psíquicos devastadores. Os estressores mais mencionados são: baixo salários ( 7 vezes), nenhum "feedback" relativo a desempenho ( 7 vezes); sobrecarga de trabalho 
(6 vezes); cortes no orçamento (6 vezes); lidar com os usuários (5 vezes); supervisão incompetente (5 vezes); nenhuma oportunidade para progredir (4 vezes); nenhum reconhecimento pelo trabalho desenvolvido (4 vezes); e expectativas que não condizem com a realidade (4 vezes).

Esta pequena lista demonstra que a literatura sugere que o ambiente de trabalho é um dos principais responsáveis pelo estresse entre bibliotecários. OSTLER \& OON (9:590) afirmam que estresse não é inerente, mas sim "o ambiente de trabalho, ou como os indivíduos interpretam seu ambiente de trabalho - isto sim, uma fonte principal de estresse." NEVILLE (10:243) enfatiza que situações de estresse no trabalho são "caracterizadas pela percepção do indivíduo com relação a eventos não desejados, o montante de avaliações, a capacidade do supervisor de julgar o desempenho, o lapso de tempo entre o desempenho e a ocorrência dos resultados, o grau de dificuldade das tarefas e a incerteza quanto ao sucesso." Estes fatores são amplificados por prazos irrealísticos, expectativas que não condizem com a realidade, e pela atmosfera política da organização.

\section{ESTRATÉGIAS PARA LIDAR COM ESTRESSE}

Estratégias de gerenciar eventos estressantes é uma área de investigação que precisa ser tratada de forma mais integral pelos estudiosos da ciência da informação. A literatura existente é tendenciosa com relação a estratégias que objetivam amenizar os efeitos negativos e nocivos do estresse. Apenas alguns estudos sugerem intervenções que resultarão em melhoria de desempenho por parte dos profissionais e funcionários.

BUBE (11:8) define estratégia como sendo "um esforço ou ação para gerenciar ou controlar imposições internas e ambientais e conflitos que excedem os recursos e potencial de um indivíduo." As estratégias de gerenciar estresse mais citadas na literatura são: apoio social e grupos de comunicação; meditação e relaxação; exercícios; dieta apropriada; estabelecer metas exequíveis; treinamento; manter um estado de alerta com relação ao problema; possuir um hobby; tirar férias; projetos de enriquecimento de trabalho; mudança organizacional; meditação transcendental, e assim por diante.
Vários estudiosos dividem estas medidas em segmentos diferentes. JOBSON (12) diferenciou entre estratégias pessoais e administrativas; WALSH (13) em estratégias organizacionais e interpessoais; RUDOLPH (14) em auto-ajuda, programas internos e externos; TODARO (15) em profissionais e pessoais; GROSSER (16) distinguiu as estratégias pessoais da organizacionais e enfatiza a reestruturação dos trabalhos e um maior controle das estratégias de gerenciar estresse; ELLIOT \& SMITH (17) sugerem que o indivíduo deve se manter cônscio de sua própria vulnerabilidade, conhecer suas fraquezas e potencialidades, controlar sua tendência de ser perfeccionista, aprender a ser flexível, e não permitir que o trabalho seja a parte mais importante de sua vida. Como gerente, o indivíduo precisa examinar o ambiente de trabalho de seus funcionários, analisar a estrutura organizacional, permitir participação na determinação de metas e processo de tomada de decisão e desenvolver técnicas de entrevista que permitam avaliar a habilidade pessoal do indivíduo para lidar com estresse."

A principal limitação destas estratégias sugeridas na literatura da área de ciência da informação é que em sua maioria elas alteram emoções para diminuir os danos psicológicos já existentes, levando a uma melhoria de sentimentos e um maior senso de satisfação apenas. As estratégias sugeridas por BUNGE (18) para lidar com sobrecarga de trabalho (estressor) é um exemplo típico:

1) ESTRESSOR. Nunca ver o trabalho terminado (senso de não estar cumprindo com as obrigações).

ESTRATÉGIA: Manter controle e estatística de tudo que se fizer.

CONCLUSÂO: A frustração de ver muito trabalho pela frente a ser realizado e a ansiedade de ver tudo pronto pode ser equilibrado por um sentimento de realização e orgulho por tudo o que já foi produzido daquele montante inicial.

2) ESTRESSOR: Filas de usuários esperando por atendimento no balcão de referência.

ESTRATÉGIA: Desenvolver habilidades de se concentrar em um único usuário, utilizando técnicas para se acalmar;

CONCLUSÃO: Se sentirá melhor em saber que ajudou mais um usuário.

Abordagens semelhantes a estas apresentadas por BUBE podem ser encontradas 
no estudo de HAACK et al. (19:48). Parece que estes autores aceitam como regra geral que o ambiente de trabalho esteja naturalmente impregnado de conflito. A sugestão deles de que "para que se possa evitar o potencial de estresse, o trabalhador precisa ser capaz de lidar com os sentimentos que acompanham conflitos..." não está direcionada a evitar o problema (estressor), mas apenas a lidar com a existência de um resultado negativo; - neste caso, o estresse.

Facilmente se percebe que em ambos os casos, as "estratégias ou medidas" adotadas não eliminam a fonte de estresse ou o estressor, mas apenas ajuda o indivíduo a lidar emocionalmente com os resultados negativos de uma situação sempre presente. Sendo que o problema não foi eliminado e as estratégias sugeridas não são capazes de evitar a ocorrência destes, as estratégias não afetarão e nem contribuirão para uma melhoria no desempenho ou produtividade. Nenhum resultado positivo resultará destas medidas. Elas apenas ajudarão o indivíduo a lidar com o revés emocional provocado pela situação ou problema.

Embora isto seja necessário e importante, resultados mais duradouros e conseqüências mais desejáveis seria aplicar intervenções voltadas a evitar a ocorrência de eventos e situações estressantes, que possibilitariam ao indivíduo, livre de barreiras emocionais, melhorar seu desempenho. Mas uma limitação irrefutável da literatura da área é o fato de que quase todas as pesquisas ali relatadas se concentram em "como lidar com as consequências negativas causadas pelo estresse", ou seja, estratégias de dimensões terciárias. As medidas são efetuadas para reduzir os danos físicos e psíquicos causados por estresse.

Alguns estudos sugerem estratégias de dimensão secundária, ou seja, intervenções voltadas para alcançar respostas que controlam o significado da experiência estressante após a ocorrência do evento estressor, mas antes do surgimento de danos severos. No entanto, pouquíssimos trabalhos abordam estratégias da dimensão primária. As estratégias desta dimensão são intervenções que objetivam alcançar respostas que mudarão a situação estressante ou as experiências estressantes provocadas por uma determinada situação. As medidas adotadas evitam a ocorrência de estresse. Muitas destas medidas neste nível produzirão resultados positivos e melhoria no desempenho porque eliminam a fontes de estresse no ambiente de trabalho.
BUBE (20:9) sugere que uma reestruturação de tarefas levará a um aumento de produtividade e NEAL (21) recomenda que para reduzir estresse no ambiente de trabalho seria necessário reestruturar as tarefas, adotar exigências realísticas e melhorar o sistema de comunicação, medidas estas, que podem contribuir para a eliminação de situações de risco e aumentar a produtividade.

O trabalho mais importante apresentado na literatura que enfatiza especificamente estratégias da dimensão primária é a investigação de NEVILLE (22). O artigo sugere mudanças na estrutura tradicional das bibliotecas - daquela que enfatiza as funções tradicionais voltadas para a organização de materiais para uma mais apropriada para servir seus clientes, refletindo uma mudança de ênfase.

Uma análise dos processos adotados nos setores das unidades de informação poderia levar em consideração as funções essencialmente ligadas a serviços aos usuários. Ao se analisar a estrutura organizacional através das categorias de funções voltadas aos usuários, os setores poderão rever e repensar as responsabilidades dos funcionários e profissionais com relação aos serviços prestados e identificar obstáculos que impedem um melhor atendimento às necessidades da comunidade usuária. Ao se aplicar as categorias destas funções voltadas aos usuários como uma abordagem de reengenharia organizacional, é também oferecida uma oportunidade para o gerente analisar a estrutura da unidade de informação do ponto de vista do cliente.

Muitos gerentes têm estruturado o fluxo de atividades, enfatizando as demandas de funções orientadas para a organização de materiais. Quando este for o caso, os mecanismos de retro-alimentação não comunicam eficazmente ao pessoal as responsabilidades envolvidas nas funções que enfatizam os serviços aos clientes.

As unidades de informação da próxima década precisam reconhecer a natureza da mudança de uma estrutura voltada para a organização de materiais e de uma voltada para oferecer serviços aos usuários. As unidades de informação precisam de uma reengenharia em sua estrutura organizacional para viabilizar esta mudança, pois apenas assim é que as condições de estresse que contribuem para a frustração e mal desempenho oriundas de uma estrutura distorcida e não condizente com sua real missão 
poderão ser transformadas em condições no ambiente organizacional que levarão à uma maior produtividade e melhor desempenho.

\section{CONCLUSÃO}

Já em 1975 a literatura enfatizava o papel da estrutura como fonte de estresse. Em um estudo voltado para determinar a relação entre a estrutura organizacional e a satisfação no trabalho, ansiedade/estresse e desempenho, IVANCEVICH \& DONNELLY (23:273) afirmam que "a ansiedade e estresse não produtivos foram reduzidos quando um determinado tipo de estrutura era adotada. Identificou-se que uma estrutura mais horizontalizada (achatada) se associava à maior produtividade e maior satisfação no trabalho mais que outros tipos de estruturas, descritas como sendo "médias" e "altas".

FISHER (23:231) concluiu que "independente de como os indivíduos modifiquem seus comportamentos, se a estrutura organizacional está causando ansiedade nos funcionários, a situação não será melhorada até que mudanças sejam realizadas em níveis mais amplos."

Portanto, é bem compreensível que se a unidade de informação não articular sua missão de serviço através de uma estrutura adequada criará ambigüidades organizacionais e um ambiente inapropriado para o desenvolvimento de atividades de atendimento ao usuário e, conseqüentemente, estresse.

Treinar o indivíduo para desenvolver estratégias pessoais para lidar com estresse fornecerá alívio temporário de certos aspectos de frustração no trabalho, mas uma reengenharia da estrutura organizacional implica em soluções mais permanentes aos problemas que afetam tanto o indivíduo quanto a organização.

Como gerente de unidade de informação, será necessário analisar as condições de trabalho, permitir participação no estabelecimento de metas e tomada de decisão e delinear uma estrutura voltada para serviços. A adoção de intervenções de dimensão primária como estas contribuirá para evitar a ocorrência de muitos eventos estressantes e levará a organização a atingir níveis de desempenho cada vez maiores, proporcionando assim um ambiente mais produtivo, onde os indivíduos trabalharão livres dos transtornos mentais e físicos causados por estresse oriundos do ambiente de trabalho.

\section{REFERÊNCIAS}

1. SEYLE, H. The stress of life. New York: McGraw Hill, 1976.

2. BERKHOUT, J. Psychophysiological stress. In: DeGREEN, K. B. Systems psychology. New York : McGraw Hill, 1970.

3. BUBE, J. L. Stress in the library environment. Technicalities, v.7, p.5-7, 1985.

4. GROSSER, K. Stress and stress management: Part I, Physiological aspects of stress. LASIE, v.15, n.5, p.2-23, 1985.

5. FREUDENBERGER, H. J., RICHELSON, G. Burnout: the high cost of high achievement. New York, 1980.

6. OLSGAARD, J. N., SUMMERS, F. W. Sources of job-related tension among administrators and faculty. JELIS, v.27, n.4, p.90-99, 1986.

7. HODGES, Julie E. Stress in the library. Library Association Record, n.92, p.751-754, 1990.

8. BUNGE, C. Staff relate sources of stress and satisfaction. Library Journal, v.112, n.15, p.47-51, 1987.

9. OSTLER, Larry J., OON, Jin Teik. Stress analysis: a case study. College \& Research Library News, n.50, p.587-590, 1989.

10. NEVILLE, S. H. Job stress and burnout: occupational hazards for services staff. College \& Research Libraries, v.42, n.3, p.242-247, 1981.

11. BUBE, J. L. Stress in the library environment. p.49.

12. JOBSON, B. S. Burnout: a problem for library managers. Southeastern Librarian, v.32, n.2, p.38-40, 1984.

13. WALSH, J. A. Burnout: the brothers grim. Illinois Libraries, v.64, n.10, p.1149-53, 1982.

14. RUDOLPH, B. Librarian burn-out. Library Journal, v.107, n.19, p.2048-2051, 1981.

15. TODARO, J. R. Job burnout: it's time we had a telethon. Illinois Libraries, v.64, n.10, p.1153-1157, 1982.

16. GROSSER, K. Stress and stress management: Part II/IV. A literature review. LASIE, v.16, n.1, p.2-22, 1985.

17. ELLIOT, J. L., SMITH, N. M. Burnout: a look at coping with stress. School Library Media Quarterly, v.12, p.136, 141-145, 
1984.

18. BUNGE, C. Staff relate sources of stress and satisfaction. p.23.

19. HAACK, M., JONES, J. W., ROOSE, T. Occupational burnout among librarians. Drexel Library Quarterly, v.20, p.46-47, 1981.

20. BUBE, J. L. Stress in the library environment. p.9.

21. NEAL, J. G. The turnover process and the academic library. In: ADVANCES in library administration and organization. Greenwich : JAI Press, 1984.

22. NEVILLE, S. H. op. cit.

23. IVANCEVICH, J. M., DONNELLY JR., J. $H$. Relation of organizational structure to job satisfaction, anxiety-stress, and performance. Administrative Science Quarterly, v.20, p.272-280, 1975.

24. FISHER, David P. Are librarians burning out? Journal of Librarianship, v.22, p.216-235, 1990.

\section{Silas Marques de Oliveira \\ Ph.D., Professor do Departamento de Pós- Graduação em Biblioteconomia da PUCCAMP, Campinas, SP.}

\section{Title}

Reengineering and Stress in Information Units

\section{Abstract}

The person's ability to deal with stress in his/her work environment has received, comparatively, little attention from researchers of the Sciences of Information area. A pertinent literature review revealed only a few works about the topic, and most of them emphasize the negative results and the devastating psychological consequences inflicted upon the person through stressing events. Few works approach interventions that would avoid the occurrence of stressing situations, wich would lead to positive results manifested through the performance improvement and productivity increase. As the information units are service agencies, the rigid, hierarchical, functional structure does not suit the nature of their activities, causing stress in the personnel. A reengineering in their structureaiming at making them compatible with their main function will eliminate tension focuses, avoiding stress and consequently improving the work environment, thus making the best of the personnel potential.

\section{Keyworlds}

Stress - Work - Information Units

Apresentado no COBIBiii em agosto de 1995 